\title{
Introduction of the Market-Based Price Autocorrelation
}

\author{
Victor Olkhov \\ Moscow, Russia \\ victor.olkhov@gmail.com \\ ORCID: 0000-0003-0944-5113
}

\begin{abstract}
This paper considers direct dependence of the market price autocorrelation on statistical moments of the market trades as a must necessary requirement. We regard market time-series of the trade value and volume as origin of price time-series. That determines dependence of the market-based averaging of price on averaging of the trade value and volume time-series. We introduce the market-based price statistical moments as functions of the statistical moments of trade value and volume. Moving average helps define the market-based price statistical moments with time-lag and introduce the price time autocorrelation as function of time-lag statistical moments of the trade value and volume. Statistical moments of the market trade value and volume are determined by conventional frequency-based probability measures. However, the price statistical moments and the price autocorrelation in particular are determined by the market-based probability measure that differs from the conventional frequency-based price probability. That distinction leads to different treatments of the price autocorrelation via market-based and frequency-based approach. To assess market dependence of price statistical moments and price autocorrelation one should revise results founded on frequency-based approach.
\end{abstract}

Keywords : asset pricing, price probability, autocorrelation, market trades JEL: G12

This research received no support, specific grant or financial assistance from funding agencies in the public, commercial or nonprofit sectors. We welcome funding our studies. 


\section{Introduction}

Predictions of the asset price are one of the most demanded market problems. Models of market price autocorrelation help deliver reasonable forecasts of future price trends and could increase returns. Financial models use market data records that present time-series of the trade value, volume and price. Analysis of time-series that describe market trade volume and price, economic or financial variables those impact or depend on market trading, determines major part of econometrics and delivers ground for the theoretical description of economics and finance. Irregular behavior of time-series records requires development of time-series analysis and (Woodward, Gray, and Elliott, 1917; Davis, 1941; Anderson, 1971; Cochrane, 2005; Montgomery, Jennings and Kulahci, 2008) present only small part of these important studies. Assessments of time-series price correlations are one of primer and obvious way to uncover internal laws and relations of price evolution and variations.

Price autocorrelation problem is a part of more general problem of time-series correlations of economic and financial variables. Economic correlations are discussed in numerous studies (King, 1917; Kendall and Hill, 1953; Friedman, 1962; Fama, 1965; Michaely, 1971; Lo, 1987; Campbell, Grossman, and Wang, 1992; Liu et.al., 1997; Mantegna and Stanley, 2000; Plerou et.al., 2000; Goetzmann, Li and Rouwenhorst, 2001; Llorente et.al., 2001; Andersen et.al., 2005; Andersen et.al., 2006; Quinn and Voth, 2008; Diebold and Strasser, 2010; Lind and Ramondo, 2018).

We are not able present any in-depth review of current state of correlation studies, but outline only few issues. Many researchers study the market price correlations: Fama (1965) and Lo (1987), Liu et.al. (1997) and Plerou et.al. (2000), Goetzmann, Li and Rouwenhorst (2001) and Quinn and Voth (2008) describe different issues and scales of the market price timeseries correlations. Karpoff, (1987) and Campbell, Grossman, and Wang (1992) model relations between stock market trading volume, price change and autocorrelations of daily stock index return. In this paper we consider the price autocorrelation problem taking into account one simple and obvious statement. All time-series delivers initial records of economic variable's data that, as usual, show highly irregular dynamics. However, the assessments of the mean properties, volatility or correlation of time-series primarily should be determined by the economic meaning of the particular variable. The time-series analysis regardless economic meaning of the particular economic or financial variable, may lead to results with poor economic sense and cause negative, loss-making trade decisions.

In our paper we consider market-based approach to the price autocorrelation problem as a necessary condition that highlights the impact of the market trading on price correlations. We 
consider the market price time-series as result of market trade value and volume time-series and consider price autocorrelation as result of autocorrelations of the market trade value and volume. Market trade records deliver irregular time-series and any reasonable description of market dynamics should operate with data smoothed or averaged during some time interval $\triangle$. Market trade dynamics delivers ground for macroeconomic modelling and forecasting. On the other hand, market trade prospects heavily relay on predictions of asset price, volatility and correlations. Any asset pricing model as well as description of market correlations are based on particular averaging procedure of initial time-series during certain time interval $\Delta$. However, price averaging procedure can't be determined by price time-series alone. We state that price averaging procedure should follow relations between trade value, volume and price. In some extent this paper develops results presented in (Olkhov, 2020; 2021a-2021d). We consider market trades performed at moment $t_{i}$ by their trade value $C\left(t_{i}\right)$ and trade volume $U(t)$ as origin of market price $p\left(t_{i}\right)$ determined by trivial relations (1.1):

$$
C\left(t_{i}\right)=p\left(t_{i}\right) U\left(t_{i}\right)
$$

Relations (1.1) define any single trade at moment $t_{i}$ and we see no obstacles that prevent use (1.1) as ground for description of the asset price statistical moments and asset price autocorrelation. We describe asset price $p\left(t_{i}\right)$ autocorrelation as function of statistical moments of the market trade value $C\left(t_{i}\right)$ and volume $U\left(t_{i}\right)(1.1)$.

Trade value $C\left(t_{i}\right)$ and volume $U\left(t_{i}\right)$ are additive variables. Sum of several trade values has meaning of aggregate value and sum of trade volumes has meaning of aggregate trade volume. However, sum of prices doesn't have meaning of aggregate price. To define aggregate price one should take ratio of aggregate value to aggregate volume. These trivial considerations help distinguish additive, like trade value and volume, and non-additive variables, like price or returns. We state that descriptions of additive economic variables establish ground for modelling non-additive variables. We consider reasons that justify taking additive economic variables like trade value and volume as primary issues that define regular or random behavior of non-additive economic variables like price in (Olkhov, 2016a-2019b). We repeat that, ratio of collective additive variables defines collective non-additive variables. For example, sum of value $C(t)$ and volume $U(t)$ of all trades performed during certain averaging time interval $\Delta$ define mean price $p(t)$ during that interval $\Delta$ similar to $(1.1)$ as

$$
C(t)=p(t) U(t)
$$

It is obvious, that the mean price $p(t)$ (1.2) of trades performed during the interval $\Delta$ with total trade value $C(t)$ and volume $U(t)$ determined by (1.2-1.4)

$$
C(t)=\sum_{i=1}^{N} C\left(t_{i}\right) ; \quad U(t)=\sum_{i=1}^{N} U\left(t_{i}\right)
$$




$$
\begin{gathered}
t_{1}=t-\frac{\Delta}{2} ; t_{i} \in\left[t-\frac{\Delta}{2} ; t+\frac{\Delta}{2}\right] ; i=1, \ldots N \\
p(t)=\frac{1}{U(t)} \sum_{i=1}^{N} p\left(t_{i}\right) U\left(t_{i}\right)=\frac{C(t)}{U(t)}
\end{gathered}
$$

coincides with well-known Volume Weighted Average Price (VWAP) $p(t)$ (1.5) introduced 30 years ago (Berkowitz et.al., 1988; Buryak and Guo, 2014; Busseti and Boyd, 2015). In our paper we consider the statistical moments and autocorrelation of the market price $p(t)$ using that single simple statement: properties of additive economic variables, as trade value and volume, determine properties of non-additive variable, as price $(1.1 ; 1.2)$.

We assume that readers are familiar enough with primary notions of the probability theory, statistical moments, characteristic function and etc.

\section{Market-based price statistical moments}

Let us consider time-series of the market trade value $C\left(t_{i}\right)$, volume $U\left(t_{i}\right)$ and price $p\left(t_{i}\right)$ in time points $t_{i}$. For simplicity assume that time-series are determined at $t_{i}(2.1)$ :

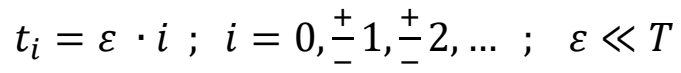

and time scale $\varepsilon(2.1)$ is much less than time horizon $T$ of our problem. Initial time scale $\varepsilon$ (2.1) determines initial time-division, the min time shift of the time-series. The values of time-series can be very irregular and to operate them on time horizon $T$ one should smooth or average initial time-series. Let us select the averaging time interval $\Delta$ and transit the time divisions of our model from time scale $\varepsilon$ to time scale $\Delta$. To do that let take $\Delta$ as:

$$
\begin{gathered}
\Delta=N \cdot \varepsilon \quad ; \quad N=2 n+1 ; \varepsilon \ll \Delta \ll T \\
t_{k}=\Delta \cdot k \quad ; \quad \Delta_{k}=\left[t_{k}-\frac{\Delta}{2} ; t_{k}+\frac{\Delta}{2}\right] \quad ; \quad k=0, \underset{-}{ \pm} 1, \frac{ \pm}{-} 2, \ldots
\end{gathered}
$$

In simple words, we divide time line by averaging intervals $\Delta_{k}(2.3)$ and replace initial timeseries $t_{i}(2.1)$ by time-series at $t_{k}(2.3)$ with time scale $\Delta \gg \varepsilon$.

The frequency-based probability approach is the most conventional way to asses mean price $p(t ; 1)$ during the interval $\Delta$. The standard interpretation of the price $p$ probability "is based on the probabilistic approach and using A. N. Kolmogorov's axiomatic of probability theory, which is generally accepted now" (Shiryaev, 1999). Assume that there are $N$ trades during the interval $\Delta$ and there are $n(p)$ trades with price $p$. Then, probability $P(p)=n(p) / N$ of price $p$ is proportional to the frequency $n(p)$ of events - frequency $n(p)$ of trades at price $p$. These common and simple relations define frequency-based probability $P(p)$ and define mean price $p(t ; 1)$ and price $n$-th statistical moment $p(t ; n)$ during the interval $\Delta$ as:

$$
\begin{gathered}
p(t ; 1)=\sum P(p) p=\frac{1}{N} \sum_{i=1}^{N} p\left(t_{i}\right) ; p(t ; n)=\sum P(p) p^{n}=\frac{1}{N} \sum_{i=1}^{N} p^{n}\left(t_{i}\right) \\
t_{1}=t-\frac{\Delta}{2}
\end{gathered}
$$


For simplicity we omit index $k$ at moment $t$. Similar frequency-based probability considerations define $n$-th statistical moments of the market trade value $C(t ; n)$ and volume $U(t ; n)$ during the interval $\Delta$ :

$$
C(t ; n)=\frac{1}{N} \sum_{i=1}^{N} C^{n}\left(t_{i}\right) \quad ; \quad U(t ; n)=\frac{1}{N} \sum_{i=1}^{N} U^{n}\left(t_{i}\right)
$$

Assessment of $n$-th statistical moments (2.5) approximate treatment of trade value $C\left(t_{i}\right)$ and volume $U\left(t_{i}\right)$ as random variables during the interval $\Delta$. For $n=1$ statistical moments $C(t ; 1)$ and $U(t ; 1)$ describe mean trade value and volume at moment $t$ averaged during the interval $\Delta$. However, we remark that price is not self-sufficient process, but is the result of market trades, determined by its trade value and volume as (1.1). Thus price probability and price averaging procedure primarily should follow the economic rationally and market content of the price statistical moments. Standard frequency-based probability perfectly describes statistical moments of the additive variables as trade value and volume. However, to keep economic sense, market meaning of price statistical moments, one should use market-based approach to description of price random properties.

It is obvious, that relations (1.1) prohibit usage of independent definitions of averaging procedures for all three variables - trade value $C\left(t_{i}\right)$, volume $U\left(t_{i}\right)$ and price $p\left(t_{i}\right)$. Averaging procedures of the trade value $C\left(t_{i}\right)$ and volume $U\left(t_{i}\right)$ determine averaging procedure of the market price $p\left(t_{i}\right)$ (1.1). To keep economic meaning of price $n$-th statistical moments $p(t ; n)$ they should be determined by statistical properties of the trade value and volume. In other words: price $n$-th statistical moments $p(t ; n)$ should match consequences of (1.1) determined by statistical moments of trade value $C(t ; n)$ and volume $U(t ; n)$. To derive these relations let consider (1.1) of the single market trade at time $t_{i}$ and take it's $n$-th power:

$$
C^{n}\left(t_{i}\right)=p^{n}\left(t_{i}\right) U^{n}\left(t_{i}\right)
$$

Now let average (2.6) and assume that time series of price $n$-th power $p^{n}\left(t_{i}\right)$ do not correlate with $n$-th power of trade volume $U^{n}\left(t_{i}\right)$. Actually, definition of widely used during last 30 years VWAP (Berkowitz et.al., 1988; Buryak and Guo, 2014; Busseti and Boyd, 2015) also uses assumption of no correlations between price $p\left(t_{i}\right)$ and volume $U\left(t_{i}\right)$. Then obtain

$$
\begin{gathered}
<C^{n}\left(t_{i}\right)>=<p^{n}\left(t_{i}\right) U^{n}\left(t_{i}\right)>=<p^{n}\left(t_{i}\right)><U^{n}\left(t_{i}\right)> \\
C(t ; n)=<C^{n}\left(t_{i}\right)>; U(t ; n)=<U^{n}\left(t_{i}\right)>; \quad p(t ; n)=<p^{n}\left(t_{i}\right)>
\end{gathered}
$$

We use $<\ldots>$ to denote the averaging procedure. Assumption (2.7) defines market-based $n$-th statistical moments of the price $p(t ; n)$ as functions of statistical moments the trade value $C(t ; n)$ and volume $U(t ; n)$ determined by frequency-based probabilities (2.5) and (2.7) takes form:

$$
C(t ; n)=p(t ; n) U(t ; n)
$$


It should be emphasized that market-based approach to definition of price statistical moments $p(t ; n)(2.7 ; 2.8)$ gives relations that are completely different from price statistical moments determined by the frequency-based probability (2.4). The only case when (2.4) coincides with (2.8) - when all members of trade volume time series equal unit: $U\left(t_{i}\right)=1, i=1, \ldots$. However that case does not describe real market trade.

The assumption of no correlations (2.7) does not mean that time-series of price $p\left(t_{i}\right)$ and trade volume $U\left(t_{i}\right)$ statistically independent. One may assess correlations between $n$-th power of price $p^{n}\left(t_{i}\right)$ and $m$-th power of trade volume $U^{m}\left(t_{i}\right)$ for $n \neq m$.

However, our remark that VWAP implies no correlations between price and volume timeseries might wonder those, who are familiar with numerous studies on price-volume relations (Karpoff, 1987; Gallant, Rossi and Tauchen, 1992; Odean, 1998; Gopikrishnan, et.al, 2000; Podobnik, et.al, 2009; DeFusco, Nathanson and Zwick, 2017). We underline that the origin of that contradiction between their results and our statement of no price-volume correlations is determined by different treatment of price averaging procedures. That illustrates distinction between the conventional frequency-based approach to price probability (2.4) and our market-based treatment of price probability $(2.7 ; 2.8)$ determined by statistical moments of the trade value and volume.

Relations (2.8) introduce price volatility $\sigma^{2}(p)$ (2.9) at time $t$ as function of trade value and volume statistical moments $(2.5 ; 2.8)$ :

$$
\sigma_{p}^{2}(t)=p(t ; 2)-p^{2}(t ; 1)=\frac{C(t ; 2)}{U(t ; 2)}-\frac{C^{2}(t ; 1)}{U^{2}(t ; 1)}
$$

The set of price $n$-th statistical moments (2.8) for all $n=1, \ldots$ for the averaging interval $\Delta$ determines (Klyatskin 2005; 2015) Taylor series of the price characteristic function $F(t ; x)$ :

$$
F(t ; x)=1+\sum_{i=1}^{\infty} \frac{i^{n}}{n !} p(t ; n) x^{n}=1+\sum_{i=1}^{\infty} \frac{i^{n}}{n !} \frac{C(t ; n)}{U(t ; n)} x^{n}
$$

Characteristic function (Shephard 1991; Shiryaev 1999) $F(t ; x)$ (2.10) completely describes properties of market price as a random variable at time $t$ during the interval $\Delta$. On the other hand, the characteristic function $F(t ; x)(2.10)$ is completely determined by the statistical moments of the trade value $C(t ; n)$ and volume $U(t ; n)(2.5)$ that are results of the frequencybased probability. However, the price characteristic function $F(t ; x)$ (2.10) and the price statistical moments $p(t ; n)(2.8)$ are determined by probability measure that is different from the frequency-based probability. We refer (Olkhov, 2021a-2021d) for further details.

Above considerations describe the price statistical moments (2.8) at time $t_{k}$ multiple by interval $\Delta(2.2 ; 2.3)$. Thus any attempt to describe the price autocorrelation within the same 
averaging procedure can assess autocorrelation for time-lag scale multiple of $\Delta$. That may be too large and to reduce time-lag one should use approximation based on moving average.

\section{Moving average and market-based price autocorrelation}

Moving average is a well-know tool for time-series analysis (Anderson, 1977, Cochrane, 2005; Montgomery, Jennings and Kulahci, 2008; Woodward, Gray and Elliott, 1917). In simple words, moving average reproduces above averaging procedure during the interval $\Delta$ $(2.2 ; 2.3)$ but central time points $t_{k}$ of the interval $\Delta_{k}(2.3)$ are taken multiple of the different time scale $l$ as:

$$
t_{k}=l \cdot k \quad ; \quad \Delta_{k}=\left[t_{k}-\frac{\Delta}{2} ; t_{k}+\frac{\Delta}{2}\right] \quad ; \varepsilon \leq l \leq \Delta ; \quad k=0, \pm \frac{ \pm}{-} 1, \pm 2, \ldots
$$

Averaging "window" $\Delta$ moves with time-lag $l$ and for simplicity one can take $l$ as multiple of $\varepsilon$. Thus one obtains almost the same mean values of the time-series averaged during the interval $\Delta$ but with time-lag $l$ that can be less then $\Delta$ (3.1). For $l=\Delta$ one obtains the above case $(2.2$; 2.3). However, moving average allows assess time-series price autocorrelations with more accuracy.

To assess time correlation consider the single trade relations (1.1). Let us consider two trades (1.1) with time-lag multiple of $l$ and take their product:

$$
C\left(t_{i}\right) C\left(t_{i}+l m\right)=p\left(t_{i}\right) p\left(t_{i}+\operatorname{lm}\right) U\left(t_{i}\right) U\left(t_{i}+l m\right) ; m=0,1,2 \ldots
$$

Relations (3.2) are simple product of two trades (1.1) at time points $t_{i}$ and $t_{i}+l m$ and are starting expression for definition of market-based price autocorrelation with time-lag $l m$. Indeed, consider 2- $d$ statistical moment of the trade value (3.3) and volume (3.4) at moment $t_{k}$ and the time-lag $l m$

$$
\begin{gathered}
C\left(t_{k}, t_{k}+l m\right)=\frac{1}{N} \sum_{i=1}^{N} C\left(t_{i}\right) C\left(t_{i}+l m\right) ; t_{1}=t_{k}-\Delta / 2 \\
U\left(t_{k}, t_{k}+l m\right)=\frac{1}{N} \sum_{i=1}^{N} U\left(t_{i}\right) U\left(t_{i}+l m\right) \\
C\left(t_{k}, t_{k}+\operatorname{lm} ; \boldsymbol{x}\right)=<C\left(t_{i}\right) C\left(t_{i}+\operatorname{lm}\right)>; U\left(t_{k}, t_{k}+\operatorname{lm} ; \boldsymbol{x}\right)=<U\left(t_{i}\right) U\left(t_{i}+\operatorname{lm}\right)>
\end{gathered}
$$

Now, similar to Sec.2, we assume no correlations between price product in (3.2) and trade volume product (3.6):

$$
<p\left(t_{i}\right) p\left(t_{i}+\operatorname{lm}\right) U\left(t_{i}\right) U\left(t_{i}+\operatorname{lm}\right)>=<p\left(t_{i}\right) p\left(t_{i}+\operatorname{lm}\right)><U\left(t_{i}\right) U\left(t_{i}+\operatorname{lm}\right)>(3.6
$$

For $m=0$ assumption (3.6) is consistent with (2.7) for $n=2$. Taking (3.3-3.6) we define price 2- $d$ statistical moment $p\left(t_{k}, t_{k}+\operatorname{lm}\right)$ (3.7) with time-lag $\operatorname{lm}$ similar to (2.8):

$$
\begin{aligned}
& C\left(t_{k}, t_{k}+l m\right)=p\left(t_{k}, t_{k}+\operatorname{lm}\right) U\left(t_{k}, t_{k}+l m\right) \\
& p\left(t_{k}, t_{k}+l m\right)=<p\left(t_{i}\right) p\left(t_{i}+l m\right)>
\end{aligned}
$$


Relations $(2.5 ; 2.8)$ and (3.3-3.8) define autocorrelations of the trade value $B_{C}(t, t+\tau)$ (3.9), volume $B_{U}(t, t+\tau)$ (3.10) and price $B_{p}(t, t+\tau)$ (3.11) at time $t$ with time-lag $\tau=l m$. We omit index $k$ for simplicity.

$$
\begin{aligned}
& B_{C}(t, t+\tau)=C(t, t+\tau)-C(t ; 1) C(t+\tau ; 1) \\
& B_{U}(t, t+\tau)=U(t, t+\tau)-U(t ; 1) U(t+\tau ; 1) \\
& B_{p}(t, t+\tau)=p(t, t+\tau)-p(t ; 1) p(t+\tau ; 1)
\end{aligned}
$$

We underline that autocorrelations of trade value $B_{C}(t, t+\tau)$ (3.9) and volume $B_{U}(t, t+\tau)$ (3.10) are determined by the conventional frequency-based probability $(3.3 ; 3.4)$ and are in common use. However, market-based price autocorrelation $B_{p}(t, t+\tau)$ (3.11) is completely determined by statistical moments of the trade value and volume and differs from frequency-based price autocorrelation. If time-lag $\tau=0$, then, as required, price autocorrelation $B_{p}(t, t)(3.11)$ coincides with price volatility (2.9):

$$
B_{p}(t, t)=p(t ; 2)-p^{2}(t ; 1)=\sigma_{p}^{2}(t)
$$

For market-based price autocorrelation $B_{p}(t, t+\tau)$ (3.11) relations (3.9-3.10) imply that correlation scale $\tau_{p}$ of $B_{p}(t, t+\tau)$ (3.11) should be equal the max of scale $\tau_{C}$ of the trade value $B_{C}(t, t+\tau)$ and scale $\tau_{U}$ of trade volume $B_{U}(t, t+\tau)$ autocorrelations. Correlation scale determines time-lag at which autocorrelation falls to zero. Indeed, present the price autocorrelation $B_{p}(t, t+\tau)$ via statistical moments of the trade value and volume:

$$
B_{p}(t, t+\tau)=\frac{C(t, t+\tau)}{U(t, t+\tau)}-\frac{C(t ; 1)}{U(t ; 1)} \frac{C(t+\tau ; 1)}{U(t+\tau ; 1)}
$$

If $\tau_{C}$ - the scale of trade value correlation and $B_{C}\left(t, t+\tau_{C}\right)=0$ and then

$$
C\left(t, t+\tau_{C}\right)=C(t ; 1) C\left(t+\tau_{C} ; 1\right)
$$

If $\tau_{C}<\tau<\tau_{U}$, and $\tau_{U}$ - the scale of the volume autocorrelation $B_{U}\left(t, t+\tau_{U}\right)=0$ then

$$
B_{p}(t, t+\tau)=-\frac{B_{U}(t, t+\tau)}{U(t, t+\tau)} \frac{C(t ; 1) C(t+\tau ; 1)}{U(t ; 1) U(t+\tau ; 1)} ; \tau_{C}<\tau<\tau_{U}
$$

We underline that in that case for positive volume autocorrelation $B_{C}\left(t, t+\tau_{C}\right)>0$, price autocorrelation is negative $B_{p}(t, t+\tau)<0$ and vise versa. For this case the price autocorrelation $B_{p}(t, t+\tau)$ equals zero at the scale $\tau_{U}$ of the trade volume

$$
B_{U}\left(t, t+\tau_{U}\right)=0 \text { hence } B_{p}\left(t, t+\tau_{U}\right)=0
$$

Otherwise, if $\tau_{C}>\tau>\tau_{U}$, then similar to (3.12-3.15) obtain:

$$
B_{p}(t, t+\tau)=\frac{B_{C}(t, t+\tau)}{U(t ; 1) U(t+\tau ; 1)} \quad ; \quad \tau_{U}<\tau<\tau_{C}
$$

For (3.16) the price autocorrelation $B_{p}(t, t+\tau)$ has same sign as the value autocorrelation $B_{C}(t, t+\tau)$. For that case (3.16) price autocorrelation goes zero at trade value scale $\tau_{C}$ :

$$
B_{C}\left(t, t+\tau_{C}\right)=0 \text { hence } B_{p}\left(t, t+\tau_{C}\right)=0
$$


We underline that we consider price autocorrelation scales for definitions (3.2-3.11). Considerations, similar to (3.2-3.8) permit define statistical moments of the trade value, volume and price at moments $t_{<} t+\tau_{1}<\ldots<t+\tau_{n}$ as:

$$
\begin{gathered}
C\left(t, t+\tau_{1}, . . t+\tau_{n}\right)=<\prod_{k=0, . . n} C\left(t+\tau_{k}\right)>; \tau_{0}=0 \\
U\left(t, t+\tau_{1}, . . t+\tau_{n}\right)=<\prod_{k=0, . . n} U\left(t+\tau_{k}\right)> \\
p\left(t, t+\tau_{1}, . . t+\tau_{n}\right)=<\prod_{k=0, . . n} p\left(t+\tau_{k}\right)> \\
<\prod_{k=0, . . n} C\left(t+\tau_{k}\right)>=\frac{1}{N} \sum_{i=1}^{N} \prod_{k=0, . . n} C\left(t_{i}+\tau_{k}\right) ; t_{1}=t-\Delta / 2 \\
C\left(t, t+\tau_{1}, . . t+\tau_{n}\right)=p\left(t, t+\tau_{1}, . . t+\tau_{n}\right) U\left(t, t+\tau_{1}, . . t+\tau_{n}\right)
\end{gathered}
$$

Statistical moments $(3.18 ; 3.19 ; 3.22)$ describe random properties of the trade value $C(t)$, volume $U(t)$ and price $p(t)$ treated as random functions. Price statistical moments $p\left(t_{1}, . . t_{n}\right)$ (3.22) define price characteristic functional (Shephard 1991; Shiryaev 1999; Klyatskin 2005; 2015) $F[x(t)]$ as functional Taylor series similar to (2.10) as

$$
F[x(t)]=1+\sum_{i=1}^{\infty} \frac{i^{n}}{n !} \int d \xi_{1} . . d \xi_{n} \quad p\left(\xi_{1}, . . \xi_{1}\right) x\left(\xi_{1}\right) \ldots x\left(\xi_{n}\right)
$$

However, one can assess only few first price statistical moments (3.22) and should take into account that relations (3.18-3.22) give only approximations of the statistical moments that depend on amount of members of initial time-series $t_{i}$ inside the interval $\Delta$, on the lag scale $l$, on the width of the averaging interval $\Delta$ and thus integrals in (3.23) are reduced by width of the interval $\Delta$, and etc. Actually, characteristic functional (3.23) may be useful for description of the price treated as a random function (Klyatskin 2005; 2015) but one should know at least elements of functional calculus and remind above restrictions and approximations. To assess approximations of the market-based price probability that determines price statistical moments $p(t ; n)$ (2.8) one should take Fourier transforms of approximations of the characteristic function $F(x)$ (2.10) determined by first several members of the sums (2.10) (Olkhov, 2021b-c). Usage of functional calculus can help derive approximations of the price probability that describes price as a random function.

\section{Conclusion}

Market-based price statistical moments $(2.8 ; 3.7)$ and price autocorrelation $(3.11 ; 3.12)$ differs from standard frequency-based approach (Kendall and Hill, 1953; Fama, 1965; Michaely, 1971; Lo, 1987; Liu et.al., 1997; Mantegna and Stanley, 2000; Plerou et.al., 2000; Goetzmann, Li and Rouwenhorst, 2001; Quinn and Voth, 2008; Diebold and Strasser, 2010; Nava et.al, 2018). One should consider the similar market-based approach to describe statistical moments and autocorrelations of stock returns as functions of price statistical moments $(2.8 ; 3.7)$. Such market-based approach to price and return statistics illustrates firm 
dependence on random properties of market trade value and volume. That does not simplify description of price and return statistical moments and autocorrelation but maintain their economic, market meaning. Treatment of economics as a unified system with complex interrelations between its variables makes impossible studies of price trends, volatility and correlations independently from consideration of the random market trades and the economic environment. It is obvious that the unified description is too complex and different approximations should be developed. However, each particular approximation should maintain the economic meaning of the variables. Conventional frequency-based approach to statistical properties of price, returns and other non-additive variables helps study their timeseries as self-sufficient elements, simplifies the problem but the results have poor economic meaning and do not support dependence on market random properties.

However, economics is a social science and highly depends on agent's views, habits, expectations and preferences. Economic processes and market transactions in particular, mostly are governed by agent's decisions. Agents are free in their choice of preferred definitions of price statistical moments and price autocorrelation, no matter what economic meaning they have. Agents may take trade decisions on base of conventional standard frequency-based price probability and price statistical moments (2.4) or follow market-based price statistical moments and autocorrelation $(2.8 ; 3.7 ; 3.11 ; 3.12 ; 3.22)$. Agent's habits and beliefs may make frequency-based price probability treatment more preferred. However, permanent mismatch between frequency-based assessments of price statistical moments and real market outcomes may add scores in favor of the market-based description of the price statistical moments and price autocorrelation in particular. Actually, it will be more difficult methods, but times of simple solutions are far over.

The choice of the market-based treatment of the price probability, price statistical moments and price autocorrelation may require reassessments of the observed market time-series and conventional conclusions. That may deliver new vision of the market price statistical properties that should more relays on the market random behavior. Which is clear, proposed transition from frequency-based to market-based treatment of price probability and price autocorrelation will not simplify the problem. However, it may help develop approximations that will give more adequate predictions of the market reality. 


\section{References}

Anderson, T.W. (1971). The Statistical Analysis of Time-Series. Wiley\&.Sons, Inc., 1-757

Andersen, T.G., Tim Bollerslev, T., Peter F. Christoffersen, P.F. and F.X. Diebold, (2005).

Practical Volatility And Correlation Modelling For Financial Market Risk Management,

NBER, WP11069, Cambridge, MA, 1-41

Andersen, T.G., Bollerslev, T., Christoffersen, P.F., and Diebold, F.X. (2006). Volatility and

Correlation Forecasting, in G. Elliot, C.W.J. Granger, and Allan Timmermann (eds.),

Handbook of Economic Forecasting. Amsterdam: North-Holland, 778-878

Berkowitz, S.A., Dennis, E., Logue, D.E. and E.A. Jr. Noser, (1988). The Total Cost of

Transactions on the NYSE, The Journal of Finance, 43, (1), 97-112

Buryak, A. and I. Guo, (2014). Effective And Simple VWAP Options Pricing Model, Intern.

J. Theor. Applied Finance, 17, (6), 1450036, https://doi.org/10.1142/S0219024914500356

Busseti, E. and S. Boyd, (2015). Volume Weighted Average Price Optimal Execution, 1-34, arXiv:1509.08503v1

Campbell, J.Y., Grossman, S.J. and J.Wang, (1992). Trading Volume and Serial Correlation in Stock Return. NBER WP 4193, Cambridge, MA., 1-45

Cochrane, J.H. (2005). Time Series for Macroeconomics and Finance. Graduate School of

Business, Univ. Chicago, 1-136

Davis, H.T., (1941). The Analysis of Economic Time-Series. Cowles Commission for Research in Economics. The Principia Press, Inc., 1-627.

DeFusco, A.A., Nathanson, C.G. and E. Zwick, (2017). Speculative Dynamics of Prices and Volume, Cambridge, MA, NBER WP 23449, 1-74

Diebold, F.X. and G. Strasser, (2010). On The Correlation Structure Of Microstructure

Noise: A Financial Economic Approach, NBER, WP 16469, Cambridge, MA, 1-65

Fama, E.F. (1965). The Behavior of Stock-Market Prices. The Journal of Business, 38 (1), 34-105.

Friedman, M. (1962). The Interpolation of Time Series by Related Series, NBER, Cambridge, MA, Chapter 3, Correlation Methods, 14-22,

Gallant, A.R., Rossi, P.E. and G. Tauchen, (1992). Stock Prices and Volume, The Review of Financial Studies, 5(2), 199-242

Goetzmann, W.N., Li, L. and K. G. Rouwenhorst, (2001). Long-Term Global Market Correlations. NBER, WP 8612, Cambridge, MA., 1-52

Gopikrishnan, P., Pleroua, V., Liua, Y., Amarala, L.A.N., X. Gabaix, X. and H.E. Stanley, (2000). Scaling and correlation in financial time series, Physica A, 287, 362-373 
Karpoff, J.M. (1987). The Relation Between Price Changes and Trading Volume: A Survey. The Journal of Financial and Quantitative Analysis, 22 (1), 109-126

Kendall, M.G and A.B. Hill, (1953). The Analysis of Economic Time-Series-Part I: Prices. Jour. Royal Statistical Soc., Series A, 116 (1), 11-34.

King, W.I. (1917). The Correlation of Historical Economic Variables and the Misuse of Coefficients in this Connection. Publications of the American Statistical Association, 15 (120), 847-853

Klyatskin, V.I. (2005). Stochastic Equations through the Eye of the Physicist, Elsevier B.V. Klyatskin, V.I. (2015). Stochastic Equations: Theory and Applications in Acoustics, Hydrodynamics, Magnetohydrodynamics, and Radiophysics, v.1, 2, Springer, Switzerland Lind, N. and N. Ramondo, (2018). Trade With Correlation, NBER, WP 24380, Cambridge, MA, 1-64

Liu,Y., Cizeau, P., Meyer, M., Peng, C-K. and H. E. Stanley, (1997). Correlations in Economic Time Series. Physica A, 245, 437-440

Llorente, G., Michaely R., Saar, G. and J. Wang. (2001). Dynamic Volume-Return Relation of Individual Stocks. NBER, WP 8312, Cambridge, MA., 1-55

Lo, A.W., (1987). Long-term Memory in Stock Market Prices. NBER WP 2984, Cambridge, MA., 1-47

Mantegna, R.N. and H. E. Stanley, (2000). An Introduction To Econophysics. Correlations And Complexity In Finance. Cambridge Univ.Press, 1-147

Michaely, M. (1971). Appendix: A Few Experiments with Formal Correlation Analysis, 281287 in Ed. Michaely, M., The Responsiveness of Demand Policies to Balance of Payments: Postwar Patterns, NBER, Cambridge, MA.

Montgomery, D.C., Jennings, C.L. and M. Kulahci. (2008). Introduction to Time Series Analysis and Forecasting. Wiley\&.Sons, Inc., 1-469

Nava, N., Di Matteo, T. and A. Tomaso, (2018). Dynamic correlations at different timescales with empirical mode decomposition. Physica A, 502, 534-544

Olkhov,V. (2016a). On Economic space Notion, Intern. Rev. Financial Analysis. 47, 372-381

Olkhov, V. (2016b). Finance, Risk and Economic space, ACRN Oxford Journal of Finance\&Risk Perspectives, 5, 209-221

Olkhov, V., (2017). Quantitative wave model of macro-finance, International Review of Financial Analysis, 50, 143-150

Olkhov,V. (2018). How Macro Transactions Describe the Evolution and Fluctuation of Financial Variables, Int. J. Financial Stud., 6 (38), 1-19 
Olkhov, V. (2019a). Economic And Financial Transactions Govern Business Cycles. ACRN Oxford Journal of Finance\&Risk Perspectives. 8, 1-20

Olkhov,V. (2019b). Financial Variables, Market Transactions, and Expectations as Functions of Risk. Int. J. Financial Stud., 7, 66; 1-27

Olkhov, V., (2020), Classical Option Pricing and Some Steps Further. MPRA, WP105431, 1-16, https://mpra.ub.uni-muenchen.de/105431/

Olkhov, V. (2021a). Price, Volatility and the Second-Order Economic Theory. ACRN Jour. Finance \& Risk Perspectives, Sp. Issue, 18th FRAP Conference, 10, 139-165

Olkhov, V., (2021b), Three Remarks On Asset Pricing. SSRN WP3852261, 1-24. https://ssrn.com/abstract=3852261

Olkhov, V. (2021c). To VaR, or Not to VaR, That is the Question. SSRN, WP3770615, 1-14. https://ssrn.com/abstract=3770615

Olkhov, V. (2021d). Theoretical Economics and the Second-Order Economic Theory. What is it? SSRN, WP 3975585, 1-14. https://ssrn.com/abstract=3975585

Odean,T. (1998). Volume, Volatility, Price, And Profit When All Traders Are Above Average, The Journal of Finance, LIII, (6), 1887-1934

Plerou, V., Gopikrishnan, P., Rosenow, B., Amaral, L.A.N. and H. E. Stanley, (2000). Econophysics: Financial time series from a statistical physics point of view, Physica A, 279 443-456

Podobnik, B., Horvatic, D., Petersena, A.M. and H. E. Stanleya, (2009). Cross-correlations between volume change and price change, Proceed. National Academy of Sci., US (PNAC), 106 (52), 22079-22084

Quinn, D.P. and H-J. Voth, (2008). A Century of Global Equity Market Correlations. American Economic Review, 98 (2), 535-540

Shephard, N.G. (1991). From Characteristic Function to Distribution Function: A Simple Framework for the Theory. Econometric Theory, 7 (4), 519-529

Shiryaev, A.N. (1999). Essentials Of Stochastic Finance: Facts, Models, Theory. World Sc. Pub., Singapore. 1-852

Woodward, W.A., Gray, H.L. and A. C. Elliott, (1917). Applied Time Series Analysis, R. Taylor \& Francis Group, 1-752 